\title{
BIOETICA Y ORGANIZACION FUNCIONAL DE LA SOCIEDAD
}

\section{Pedro Morandé Court*}

\begin{abstract}
Resumen: El intercambio interdisciplinario de la bioética, difícil y laborioso, es el objeto de este articulo. Reflexiona, en particular, sobre dos temáticas. En primer lugar, respecto de la eventual novedad y creatividad de la bioética desde el punto de vista del pensamiento. Esta reflexión se desarrolla esencialmente en el ámbito de la filosofía, a través de una pregunta que orienta el análisis: ¿qué agrega la bioética al tradicional debate ético que se ha producido en el seno de todas las culturas? En segundo lugar, por medio de un enfoque de tipo sociológico, sobre el espacio que ocupa la bioética en una sociedad multifuncional. En esta perspectiva, este texto se interroga sobre la posibilidad de desarrollar un concepto de bioética que abarque el conjunto de la sociedad, es decir, que genere la misma legitimidad y estima en todos los sectores funcionalmente diferenciados.
\end{abstract}

Palabras clave: Bioética, sociedad, debate ético, ontología simbólica, biotecnología

\section{BIOETHICS AND SOCIETY'S FUNCTIONAL ORGANIZATION}

\begin{abstract}
Bioethics' interdisciplinary interchange, difficult and arduous, is this article's aim. First of all in relation to bioethics' fortuitous novelty and creativity from the mind's point of view. Essentially this reflection develops within philosophy's field, through a question that guides its analysis: what does bioethics add to the traditional ethical debate that has taken place in the midst of all cultures? In second place, through a sociological type of view, on the space that bioethics occupies in a multifunctional society. In this perspective, this text questions itself on the possibility of developing a bioethics concept that involves the whole society, that is, that it generates the same legitimacy and esteem in all sectors functionally differenciated.
\end{abstract}

Keywords: Bioethics, society, ethical debate, symbolic onthology, biothecnology

\section{BIOÉTICA E ORGANIZAÇÃO FUNCIONAL DA SOCIEDADE}

Resumo: O intercambio interdisciplinar da Bioética, difícil e laborioso, é o objeto desse artigo. Em particular, faz reflexão sobre duas temáticas. Em primeiro lugar, aborda a eventual novidade e criatividade da Bioética sob o ponto de vista do pensamento. Esta reflexão desenvolve-se essencialmente no âmbito da filosofia, através de uma questão: o que acrescenta a Bioética ao tradicional debate ético que tem ocorrido no seio de todas as culturas? Em segundo lugar, busca-se identificar o enfoque sociológico considerando o espaço que ocupa a Bioética numa sociedade multifuncional. Nesta perspectiva, este artigo propõe questionar a possibilidade de desenvolver um conceito de Bioética que alcance o conjunto de toda a sociedade, ou seja, que gere a mesma legitimidade e estima em todos os setores funcionalmente diferenciados.

Palavras chave: Bioética, sociedade, debate ético, ontologia simbólica, biotecnologia

\footnotetext{
* Decano de la Facultad de Ciencias Sociales, Pontificia Universidad Católica de Chile. Correspondencia: pmorande@puc.cl
} 
La constitución de la bioética como una semántica social relevante en los últimos 25 años no se ha producido ciertamente por la irrupción de alguna novedad epistemológica propia de los paradigmas éticos vigentes, sino más bien como resultado del entusiasmo y del temor suscitados por los sorprendentes avances de la biotecnología y de las ciencias de la vida. La proyección de estos nuevos saberes y tecnologías no sólo abarca el campo de la medicina y de la asistencia sanitaria, sino también el ámbito de la seguridad, del derecho, del comercio, de los modelos de desarrollo socioeconómicos y de los equilibrios medioambientales. Desde este punto de vista, la expresión bioética resulta bastante equívoca. $\mathrm{Su}$ ámbito más específico ha sido hasta ahora el relativo a la ontogénesis del individuo humano, los límites de su manipulación biogenética, la interrupción de su gestación (aborto) o la anticipación deliberada de su término natural (eutanasia). Siendo estos problemas ya en sí mismos suficientemente complejos, la discusión pública de los mismos suele estar ligada no sólo a la existencia de casos clínicos particulares o excepcionales, sino también a la adopción de determinadas opciones legislativas, de estrategias para las políticas públicas y de prioridades de inversión, tanto en el plano nacional como internacional. No hay casuística que pueda funcionar en este caso y nunca habrá tampoco suficiente información científicamente comprobada tratándose de la prevención de riesgos difíciles de estimar. Ante una situación objetivamente desconocida tienden a acentuarse las posiciones que, por una parte, abogan por la prudencia aconsejada por el beneficio de la duda y, por otra, abogan por el desarrollo sin limitación de la innovación tecnológica, confundiendo, en el extremo lo que es lícito hacer con lo que es técnicamente factible de obtener.

Habiéndose consolidado el uso de la expresión «bioética», no obstante su ambigüedad, habría que reconocer, al menos, que las potencialidades de desarrollo en el ámbito biotecnológico y de las ciencias de la vida afecta a la sociedad entera y no de modo uniforme e igualitario entre los distintos países del mundo o entre los subgrupos de una sociedad, sino de forma diferenciada, según las propias conveniencias, intereses estratégicos y posibilidades de aplicación de estos nuevos saberes e informaciones a su desarrollo respectivo. En este sentido, pareciera que la sociedad está aún muy lejos de constituir adecuadamente un debate preciso acerca de la naturaleza y objetivos de la bioética, teniendo en cuenta, pero al mismo tiempo diferenciando también, las variables geoestratégicas, políticas, económicas y demográficas involucradas en ella. Sin embargo, si no se dan pasos importantes en esta dirección, se corre el riesgo de aumentar aún más la confusión al formular con el lenguaje propio de los valores éticos absolutos e irrenunciables, aspectos referidos más bien a opciones técnico-racionales relativas al desarrollo estratégico de los países, con el resultado ya bastante extendido de relativizar el plano de los valores morales para justificar prácticas comerciales, comunicacionales o de intereses profesionales.

El Documento de Consulta de la Comisión de las Comunidades Europeas titulado «Hacia una visión estratégica de las ciencias de la vida y la biotecnología»(1) dado a conocer recientemente en su versión final, es un claro ejemplo del amplio espectro de problemas y oportunidades que suscita para la sociedad el desarrollo científico y tecnológico del conocimiento de la vida. El documento tiene el mérito de reconocer la importancia estratégica que tiene para las sociedades el dominio de este ámbito del saber. No obstante, no todos los dilemas y opciones que tienen implicancias éticas en este plano corresponden a lo que hasta ahora ha sido usualmente considerado como bioética. Si el término se ampliara para incluir todas sus implicancias sociales en las distintas 
esferas ya mencionadas, se corre el riesgo de perder la noción de cuál es el objeto sobre el que se quiere reflexionar. El expreso deseo de consultar además ampliamente a la población sobre los temas expuestos, que de la sola lectura del texto se aprecia su alta complejidad, lleva a la dificultad adicional de abrir debate sobre temas que no se comprenden suficientemente ni por los mismos especialistas, y con cuánta mayor razón por el ciudadano corriente.

Con el ánimo de contribuir al difícil intercambio interdisciplinario de ideas sobre estas importantes materias, quisiera abordar dos puntos principales: en primer lugar, en un sentido más filosófico, cuál es la novedad que presenta la bioética desde el punto de vista del desarrollo del pensamiento $\mathrm{y}$, en segundo lugar, en un sentido más sociológico, cuál podría ser el lugar de la bioética en el contexto de una sociedad multifuncional.

1. ¿Qué agrega la bioética al tradicional debate ético que se ha producido en el seno de todas las culturas? La respuesta a esta pregunta no parece evidente, puesto que la ética entendida como la reflexión acerca de la finalidad de los actos humanos y la finalidad de la vida misma incluye, por cierto, la reflexión sobre el valor y dignidad de cada vida humana que viene a la existencia y sobre la moralidad o inmoralidad de los actos que sobre ella se ejercen. Desde Aristóteles a Kant, con distintas terminologías y conceptualizaciones, el pensamiento ha reconocido que el ser humano no sólo es «algo» sino «alguien», que participa de la inteligencia del ser, para usar el lenguaje clásico, o que posee autoconciencia, para emplear el lenguaje moderno. El rasgo esencial de ambas formulaciones es que sólo él es capaz de autoposeerse, de hacer-cada-vez-suya su propia existencia, según dice la sugerente formulación de Heidegger.

Esta autoposesión de sí, que implica igualmente un saber de sí, es la base de su au- tonomía y de su responsabilidad, que lo hace capaz de comparecer como persona ante otras personas. En algunas ocasiones se ha acentuado la capacidad de su inteligencia para descubrir en su misma naturaleza una ley moral propia de su condición, en otras, se ha acentuado la capacidad de su voluntad para legislar racionalmente, es decir, conforme al principio de la universalidad de la razón, superando toda determinación circunstancial o particular. Estas ideas están en la base de nuestro moderno Estado de Derecho y de la Declaración internacional sobre los Derechos Humanos de 1948, suscrita por la mayoría de los Estados, no obstante sus diferencias culturales, étnicas, lingüísticas o religiosas. Son el fundamento también del principio negativamente formulado como «inmunidad de coacción» y positivamente formulado como «libertad de conciencia», con el preciso conjunto de libertades que de ella se derivan. Las mayores discusiones no se han producido sobre estas ideas básicas, sino más bien sobre el modo de armonizar socialmente los derechos de unos y otros, cuando por diversas circunstancias éstos entran en conflicto o competencia de intereses. La regla de oro de la moral, presente en prácticamente todas las culturas, es el principio de proporcionalidad y reciprocidad que suele formularse, negativamente, con la fórmula «no hagas a otro lo que no quieras que hagan contigo». Aplicada con prudencia o recta conciencia, con sabiduría en relación a las circunstancias particulares de cada situación, parece ser aún una norma plenamente vigente y muy cercana al sentido común de las personas y de la sociedad.

La bioética nos ha hecho tomar conciencia, sin embargo, y en ocasiones de modo dramático, que esta sólida construcción reflexiva de la ética descansa sobre ciertos presupuestos óntico-ontológicos. Particularmente, sobre el hecho de que la vida y la muerte habían sido considerados como hechos «naturales» que había que reconocer como dados y sobre los 
cuales no cabía propiamente una consideración ética. La moralidad se pronunciaba acerca de las condiciones para el desarrollo de la «vida buena», aquélla estimable y deseable para sí mismo y los demás, pero no sobre la vida misma o sobre la muerte. Ellas eran materia de investigación de las ciencias en cuanto a su descripción objetiva y en cuanto a la especificación del momento de su acontecer. Incluso hasta el día de hoy le basta al ordenamiento jurídico un certificado de la matrona que ha asistido a la parturienta o de un médico que constata el deceso. Podía, naturalmente, como en todo testimonio, producirse el fraude deliberado o la equivocación no intencional del testigo, pero para este proceder había criterios de juicio dados por el mismo cuerpo conceptual de la moral.

La novedad introducida por la biotecnología y las ciencias de la vida, antes que ética es ontológica, en la medida en que puede manipularse el proceso mismo de la generación de lo humano, al menos dentro de ciertos límites fijados por el propio avance del conocimiento científico, de tal suerte que el nacimiento y la muerte ya no pueden ser considerados como acontecimientos independientes y autónomos, sino que están sometidos también, aunque sea sólo parcialmente, a distinciones realizadas por el conocimiento y la sociedad. Ni el positivismo jurídico más extremo del siglo XIX pensó alguna vez que la existencia humana y su fin natural debían ser determinados por la voluntad legislativa de los parlamentos o de la autoridad administrativa. Aún el artículo primero de nuestro ordenamiento constitucional, como en forma equivalente el de muchos otros países, señala que las «personas nacen libres e iguales en dignidad y derechos», dando por descontado que la voluntad legislativa opera sólo después de constatar la existencia del nacido vivo y dando origen con ello a toda clase de conjeturas acerca del estatuto jurídico del embrión humano.
Pero como el propio debate lo ha demostrado, el problema de fondo tras estas formulaciones no es jurídico ni ético, aunque tenga consecuencias en estos planos, sino ontológico: ¿en qué consiste la unidad esencial de la entidad de lo humano como tal? Las ciencias empíricas no pueden tener respuesta para esta pregunta, puesto que trasciende toda condición hipotética. Cualquier respuesta desde la ciencia será necesariamente condicionada o bien, sobrepasando su competencia, caerá en un reduccionismo cientificista. Trasciende también la reflexión ética, puesto que, en el caso del embrión, no se cumplen las condiciones habitualmente definidas para el reconocimiento de un sujeto responsable de sus actos y no es posible, en consecuencia, esperar reciprocidad. El caso de la discusión sobre la legitimidad del aborto es emblemático. La ética pregunta: ¿puede considerarse al embrión como un agresor injusto?, dado que reconoce el derecho a la legítima defensa. Pero sabe, al mismo tiempo, que las condiciones del embrión humano no son aquéllas en que habitualmente tiene sentido formular esa pregunta.

La bioética obliga, en consecuencia, a replantear el vínculo entre ética y ontología, el que siempre ha existido, pero que, por diversas circunstancias y, particularmente, por el desconocimiento del proceso mismo de la procreación humana en sus primeras fases, permaneció oculto para la reflexión. Las posibilidades tecnológicas de intervención social en el surgimiento de la vida misma, han desocultado este vínculo y obligan al pensamiento a hacer su aporte. Me parece que ninguna fórmula de consenso político, cultural o social, sea a nivel nacional o internacional, por deseable y satisfactoria que pueda parecer, puede llenar este vacío, puesto que los consensos se producen en el ámbito de las opiniones o de los «puntos de vista» y de lo que se trata es 
determinar lo efectivamente real del ente humano y su unidad esencial.

¿Puede el pensamiento moderno, nacido del cartesianismo, llenar este vacío? Si seguimos el argumento de Heidegger, pareciera que no, puesto que el cartesianismo desemboca precisamente en la transformación de la naturaleza como objeto de la técnica. Quisiera citar a este respecto un iluminador párrafo de su ensayo acerca de «La frase de Nietzsche "Dios ha muerto"»: «Todo ente es ahora [se refiere a la metafísica moderna] o lo efectivamente real, en cuanto objeto, o lo eficiente en cuanto objetivación en la que se forma la objetividad del objeto. Representando, la objetivación dispone el objeto sobre el ego cogito. En este disponer se evidencia el ego como aquello que subyace a su propio hacer (el dis-poner poniendo-delante o re-presentando), esto es, se evidencia como subiectum. El sujeto es sujeto para sí mismo. La esencia de la conciencia es la autoconciencia. Por eso, todo ente es o bien objeto del sujeto o bien sujeto del sujeto. En todas partes, el ser de lo ente reside en el poner-se-ante-sí-mismo y, de esta manera, imponer-se... El mundo se convierte en objeto... Por haber sido querida a partir de la esencia del ser, la naturaleza aparece en todas partes como objeto de la técnica»(2). Si el análisis de Heidegger es correcto, habría que concluir que la entidad humana del embrión sólo podría determinarse en cuanto es objeto de la técnica, es decir, de la manipulación biotecnológica, no existiendo diferencia entre la vida humana y cualquier otro objeto de la técnica. Si la conclusión fuera correcta, no habría necesidad de elaborar ninguna bioética.

Desde el horizonte cristiano, por su parte, comienza a desarrollarse recientemente una ontología simbólica, de la cual Livio Melina es un connotado y calificado representante: «El punto de partida de esta posición es la afirma- ción de la diferencia ontológica: el ser es siempre ulterior a cualquier representación de la que es capaz la subjetividad y, por tanto, el sentido es irreductible a su objetivación. Sin embargo, éste se comunica y la forma de la comunicación es el signo, o mejor, el símbolo. De aquí nace el carácter originalmente simbólico del saber que no tiene como modalidad principal el concepto... Es típico del conocimiento simbólico interpelar a la libertad del sujeto que conoce para poder interpretar el signo. Libertad y razón están, por tanto, originalmente coimplicadas en el encuentro con el ser y en la conformidad con el significado que se entrega... El punto más agudo de la dramática interpelación a la libertad se verificará precisamente al conocer a la persona, vértice ontológico en el cual el ser se dona: ese conocimiento aparecerá siempre bajo la forma de un reconocimiento». Y agrega sobre la relación entre ontología y ética: «La ética es una dimensión que está necesariamente presente siempre; no viene después de un saber metafísico objetivo en el que la libertad no estaría implicada. Lo práctico no se deduce de lo especulativo. La situación original es, por su naturaleza, práctica: la verdad se dona en la acción. El saber de la persona está antes de la distinción entre especulativo y práctico. El ser se manifiesta en el símbolo real de la persona, como verdadero y como bueno a la vez y exige ser libremente reconocido. La ética, que tiene su momento originario en la relación con la persona del otro, se convierte en un lugar especialmente denso de la ontología.»(3) Pienso que posiciones como las aquí consignadas, y que no pueden ser exhaustivamente analizadas en este momento, constituyen un poderoso estímulo para el debate interdisciplinario, puesto que ofrecen pistas de investigación y de elaboración conceptual que permitan progresivamente superar el déficit de la reflexión de la que, en cierta medida, la misma bioética es un síntoma antes que una respuesta. 
Además de la clarificación del estatuto ontológico del embrión, un segundo aspecto sobre el que se ha mostrado la ambigüedad de la bioética se refiere a la necesidad de clarificar cuál es la vida para la que, la bioética, es una ética. ¿Tiene sentido extender el ámbito de la reflexión ética a otras formas de vida que no sean la vida humana sin establecer, al menos, una distinción entre la vida humana y las restantes formas de vida? Estas interrogantes han sido introducidas por algunos representantes de la así llamada «ecología profunda», que cuestiona el antropocentrismo de la reflexión ética, que sería, a su juicio, infundado. Me parece que cualquiera sea la respuesta que se elabore para estas preguntas, la cuestión de fondo, como ya se señaló, es ontológica antes que ética, o afecta propiamente el vínculo e integración entre ambos saberes. Un antropocentrismo fundado en el subjetivismo que denunciaba Heidegger en el párrafo ya citado, termina evidentemente en la consideración de toda vida, incluida también la humana, como un objeto determinado por la técnica. Personalmente considero muy razonable que ese tipo de antropocentrismo se ponga en tela de juicio. La creciente conciencia ecológica asumida por la humanidad nos ha puesto en guardia sobre la manipulación ilimitada de la naturaleza como mero objeto. Pero la denuncia implícita en la pregunta no puede llevar a respuestas apresuradas sobre lo que aún no se ha reflexionado suficientemente. ¿Cuál es la relación entre antropología y ontología? ¿Tiene la ontología presupuestos antropológicos? ¿Cuál es el fundamento de la afirmación de Heidegger de que el Dasein (el ser humano) es el único ser al que le va el ser en la pregunta por el ser? O en otra conocida fórmula usada por él mismo: ¿Cuál es el fundamento de que la condición óntica del Dasein (el ser humano) es ontológica? ¿Se puede escapar de esta afirmación o ir más allá de ella?
Deberán elaborarse aún las respuestas a estas preguntas. La ontología simbólica de la que hablábamos precedentemente podrá hacer también su aporte en esta dirección. Pero por ahora, no logro visualizar el fundamento de una ética aplicable a las relaciones del ser humano con los entes no humanos de la naturaleza que no tenga como punto de referencia el fenómeno humano como tal. Incluso el recurso al concepto mismo de ecología que, aparentemente, está libre de presupuestos antropológicos, en verdad no lo está, puesto que la clausura operacional que permite la distinción entre sistema y entorno o sistema y ambiente como una distinción de sentido, sólo puede producirse al nivel de la auto referencia de la conciencia psicológica o al nivel de la auto referencia del sistema de comunicación social. Los otros sistemas auto referenciales que estudian habitualmente las llamadas ciencias naturales no operan con una clausura operacional sobre la base de la selección del sentido y, por tanto, de la observación de la distinción entre sistema y medio ambiente.

2. El segundo asunto que quisiera abordar aquí, esta vez desde una perspectiva sociológica, es la ubicación de la bioética en el seno de una sociedad funcionalmente diferenciada. El Documento de Consulta ya mencionado de la Unión Europea, tiene el gran mérito, a mi juicio, de considerar el desarrollo estratégico de la biotecnología y de las ciencias de la vida en un perspectiva que trasciende el ámbito de la salud y abarca el conjunto de las actividades sociales para las cuales estas disciplinas adquieren creciente relevancia. Si se pudiera hablar de un denominador común a todas estas áreas, habría que decir que es el tradicional concepto de desarrollo, entendido esencialmente como desarrollo económico, aunque se ponga el acento también en el desarrollo humano como forma de equilibrar los crecientes desequilibrios en la distribución de las oportunidades, dado 
el diferencial de productividad de las mismas. Pero en este contexto cabe preguntarse si es posible desarrollar un concepto de bioética que abarque al conjunto de la sociedad y que encuentre en todos los sectores funcionalmente diferenciados la misma legitimidad y estima.

La moderna teoría de los sistemas sociales que opera sobre la lógica de la observación a través de la distinción entre sistema y entorno, ha llegado a la conclusión de que la diferenciación funcional en subsistemas especializados hace imposible que exista un punto de observación de la sociedad en su conjunto que pueda prescindir de las distinciones que han dado origen a los subsistemas funcionalmente diferenciados. Así, el subsistema económico sólo puede observar todos los componentes propios de su operación y aquellos que le son relevantes en el medio ambiente, desde la economía y, más específicamente, desde la codificación binaria de su operación en el binomio pagar/no pagar. Lo propio hace cada subsistema, como el de la ciencia, de la educación, de la política, etc. Si han alcanzado el nivel de la clausura operacional, y son reconocibles por lo tanto como subsistemas diferenciados de su entorno, sólo pueden interesarse por los objetos del mundo a partir de las posibilidades que le permite su código de operación. El desarrollo de la biotecnología y de las ciencias de la vida, tendrá en este plano tantas «lecturas» o modos de observación como existan subsistemas funcionalmente diferenciados. La vida, en general, y la vida humana, en particular, no escapa a esta multifuncionalidad de la observación, de modo que no puede garantizarse ni a priori ni a posteriori que la valoración que alcance una determinada tecnología o un conocimiento en el subsistema económico y en el subsistema de la ciencia, por ejemplo, se correspondan o sean comparables.
¿Puede ser efectiva en este plano la bioética si ella no incide ni en la formación ni en la operación de los códigos de los subsistemas? Me parece que la respuesta sólo puede ser negativa. En verdad, esto no vale sólo para la bioética, sino para el pensamiento reflexivo en general, aunque cuesta entenderlo por la influencia que hemos heredado del racionalismo que procede justamente de una época en que aún no se había consolidado la diferenciación funcional de las más importantes actividades sociales y existía bastante «redundancia» en el cumplimiento simultáneo de estas funciones por parte de varios o muchos actores o instituciones sociales. Pero una vez que la propia evolución social renuncia a la «redundancia» y constituye subsistemas auto referidos en su operación, ya no se puede pensar que la realidad social sea la realización histórica de modelos sociales de organización de la vida común elaborados por mentes iluminadas y omniscientes o por construcciones intelectuales colectivas, como las ideologías. Esto no significa, evidentemente, que el pensamiento no tenga ninguna influencia. No puede haber una operación de sentido (y toda comunicación lo es) que no disponga de un repertorio semántico adecuado que proporcione una sobreabundancia de referencias de sentido ulteriores. Sin embargo, existe una diferencia entre las distinciones con que se realizan las operaciones de los subsistemas sociales y que proceden de su propia codificación interna y las distinciones propias de la reflexión, que corresponden a una observación de segundo orden, es decir, a una metaobservación u observación de observadores.

Me parece que sólo en este plano es posible ubicar a la bioética en el seno de la sociedad funcionalmente diferenciada. Siguiendo a Luhmann(4), podríamos decir que éste es el plano que genéricamente reconocemos como el ámbito de la cultura, puesto que la observa- 
ción de segundo grado permite fundamentalmente dos cosas: en primer lugar, la autobservación que se estructura en la conciencia a partir de la diferenciación entre identidad-diferencia, es decir, entre auto referencia y hetero referencia y, en segundo lugar, descubrir el punto ciego presente en toda observación, debido al hecho de que, al trazar una diferencia, ningún observador puede situarse simultáneamente a los dos lados de lo diferenciado. Al descubrir el punto ciego de otros se adquiere conciencia del punto ciego propio y, de esta manera, de la necesidad de hacer comparaciones entre los distintos puntos de vista que retroalimenten la percepción de la identidad y diferencia.

¿Qué conclusión es posible sacar de la aplicación de esta distinción? Toda ética, como también la bioética, requiere una visión unitaria de lo humano capaz de trascender los códigos parciales con que operan los distintos subsistemas sociales. Tal visión unitaria la ha buscado el pensamiento en la correspondencia entre su propia unidad interna, su saber de sí, es decir, aquella sabiduría que precede y anticipa el saber de cualquier cosa, y la realidad observada, analizada, experimentada o vivida, según el énfasis puesto por las distintas corrientes filosóficas. En relación con la ética, esta correspondencia se daría por la coincidencia y mutua pertenencia de la verdad y la bondad, y se han buscado a lo largo de los siglos distintos fundamentos para justificar esta unidad. A nivel de una conciencia individual que piensa, parece razonable aceptar esta intuición esencial de la unidad del pensamiento y su correspondencia con la realidad, como una experiencia elemental irreductible e indeducible. De ello hablamos en el punto precedente en relación con el vínculo necesario entre bioética, antropología y ontología. Pero si tomamos en cuenta la reflexión sociológica y su propuesta de ubicar la reflexión no en el plano de la clausu- ra operacional de los subsistemas sociales, sino en el nivel de la observación de observadores, tendríamos que reconocer que esta visión unitaria de lo humano que toda ética necesita, no puede ser considerada como dada de antemano por la clausura operacional de la sociedad, puesto que ya vimos que los subsistemas funcionales son autónomos, sino que debe ser culturalmente construida a partir de la comparación de los puntos de vista que, observados por otros observadores, descubren el punto ciego de la observación.

Algunos han interpretado este aporte de las ciencias sociales errada o precipitadamente como valoración de la diversidad o, en algunos casos, como una suerte de derecho a la diversidad. ¿Pero cómo podría suponerse una valoración común de la diversidad y no diversa? ¿Cómo distinguir entre la valoración de la diversidad y la indiferencia al destino de aquél clasificado como diverso, precisamente porque se valora la diversidad? Los impactantes sucesos recientes (11 de septiembre de 2001) que han conmocionado al mundo muestran hasta qué punto estamos sumidos en la confusión respecto a una visión unitaria de lo humano. Desde el punto de vista sociológico, al menos, no podemos presuponer automatismos sociales de difusión del pensamiento ni volver a la tesis de la mano invisible o de la hegeliana «astucia de la razón». Nada parece sustituir al diálogo intercultural, interreligioso, interdisciplinario. Pero al mismo tiempo, el diálogo puede ser «de sordos» o «de ciegos», como se prefiera, si se pierde la conciencia de que toda observación procede de una distinción que tiene dos lados de los que uno sólo se ve, al menos que se observe el observar y se haga así manifiesta la estructura latente del mismo acto de hacer una distinción. ¿Qué ven los políticos que los economistas no pueden ver y viceversa? ¿Qué ven los cientistas sociales que los médicos no pueden ver y viceversa? Si fuese posible proceder 
de este modo sería no sólo más plausible constituir horizontalmente un espacio unitario donde se perciba la unidad de lo diferenciado, sino que se comprendería mejor también la relación entre la observación de primer orden y la de segundo orden, es decir, aquélla que se especifica por la operación de los sistemas y aquélla propia de la reflexión o de la autobservación.

Esta clarificación me resulta personalmente muy valiosa para comprender la diferencia entre tecnología y ética. Se escucha frecuentemente decir que una característica del mundo actual es que el desarrollo científico tecnológico no ha ido a la par del desarrollo de la ética, que ha permanecido deficitario, como si se tratara de dos actividades humanas que se ubican en el mismo plano y que pueden dialogar fácilmente entre sí. El supuesto implícito suele ser que se espera que el pensamiento asuma la función rectora y gobierne la sociedad y que la tecnología sea el instrumento a su servicio. Se omite considerar el hecho de que la revolución tecnológica experimentada en los últimos cincuenta años ha sido posible precisamente porque este supuesto implícito ya no tiene vigencia. Sin embargo, quienes así lo perciben, suelen pensar que, por alguna razón no siempre clarificada, simplemente se ha invertido, es decir, que ahora la tecnología gobierna y el pensamiento está a su servicio, sea para justificarla o para persuadir a la población que consuma sus productos. Las distinciones hechas en los párrafos precedentes, permiten comprender que la naturaleza de la tecnología no es tecnológica y que no se avanza mucho en su comprensión si se contrapone hombre y máquina.

La revolución social que está en la base del desarrollo tecnológico, es decir, la organización de la sociedad en subsistemas funcionalmente diferenciados de su entorno a partir de su propia clausura operacional, per- mite la equivalencia funcional del hombre y de la máquina en cuanto a la estructura de la toma de decisiones en contextos así especificados. Ambos se complementan y se potencian recíprocamente en cuanto se someten a criterios de optimización de los parámetros predeterminados si retroalimentan como información el resultado de sus propias operaciones y la alteración de las condiciones de su medioambiente. ¿Imitó la sociedad a las máquinas o las máquinas a la sociedad? No sabría decir sino sólo que ambos funcionan con el mismo protocolo en sus operaciones socialmente significativas. Esto es lo que también se quiere decir con la expresión «emergencia de la sociedad de la información». La tecnología no es así un conjunto inerme de máquinas y herramientas, sino un modo operativo de tomar decisiones que está abierto a las decisiones subsiguientes por la observación de sus cambios de estado. Esto es posible por la cuantificación de la información a través de la teoría de la probabilidad y la estadística, la que permite ser acumulada en secuencias de una extensión tal que ninguna inteligencia humana podría por sí misma descifrar.

Evidentemente, la ética no puede operar en este plano. No puede reducir la información a cantidades ni puede tampoco suponer la comparabilidad de las conductas personales o su reemplazo por equivalentes funcionales. Menos todavía la bioética, al menos en lo que se refiere al valor incomparable e inconmensurable de cada vida humana. En el plano operativo también es posible, dentro de ciertos límites, reducir la calidad a la cantidad como se observa en lo que habitualmente se llama «calidad de vida», pero que no es más que un eufemismo para el mejoramiento de los parámetros cuantitativos. Pero este procedimiento no puede extenderse al ámbito en que la razón reconoce el valor absoluto de la existencia de cada persona. La ética pertenece inexorablemente al ámbito de la conciencia y 
de la libertad humanas y si bien, como ha reconocido la tradición, puede interiorizarse de tal modo que se vuelva un hábito del sujeto virtuoso y prudente, ello no tiene una expresión equivalente en el plano social. Algunos respetados autores hablan de un ethos o también de un «núcleo ético-mítico» en el seno de las culturas, pero se trata evidentemente de conceptos analógicos que no pueden considerar el objeto que describen como una sumatoria de conciencias individuales o como una conciencia colectiva bajo la hipótesis de que la sociedad es un sujeto. Si se considera en cambio el lugar del pensamiento como el de la observación de segundo orden que es distinta de la observación operacional de primer orden y, no obstante, relacionada con ella, pienso que se obtiene una comprensión más realista del modo de inserción de la bioética en la sociedad.

Las decisiones relativas al desarrollo de la biotecnología y de las ciencias de la vida se tomarán seguramente en el futuro, como ha ocurrido también hasta ahora, en el ámbito di- ferenciado de muchos subsistemas funcionales. En algunos casos serán decisiones legislativas, en otros, políticas asumidas por la administración del Estado, en otros, inversiones privadas o públicas, nacionales o internacionales, en otros, programas de aprendizaje definidos a través de currículos escolares. Con la complejidad de la sociedad actual y su organización funcional no me resulta verosímil que pueda imponerse un criterio común en estas distintas esferas, constituidas también por puntos de observación diferentes. La bioética no puede constituirse en este nivel, puesto que para ello requeriría que la vida fuese materia propia de la operación de un subsistema funcional, lo que es un absurdo puesto que está supuesta como algo existente por todos y cada uno de los subsistemas funcionales. El aporte social que pueda hacer la bioética parece ser más bien el de reconstruir reflexivamente y en forma constante la unidad de la diferencia que impone la sociedad a la vida humana para organizar sus operaciones. En esta perspectiva, me parece que la constitución interdisciplinaria de la bioética resulta esencial.

\section{Referencias}

1. Comisión de las Comunidades Europeas. Documento de Consulta: Hacia una visión estratégica de las ciencias de la vida y la biotecnología. [Sitio en Internet] Disponible en: http://europa.eu.int/comm/biotechnology/pdf/doc_es.pdf Acceso en septiembre de 2002

2. Heidegger M. La frase de Nietzsche: Dios ha muerto. En Sendas Perdidas. Buenos Aires: Losada; 1969.

3. Melina L. Reconocer la vida. Problemas epistemológicos de la bioética. En Scola A. ed. ¿Qué es la vida? La bioética a debate. Santiago de Chile: Ediciones Universidad Católica de Chile; 1999: 79-80.

4. Luhmann N. La cultura como concepto histórico. En: Teoría de los Sistemas Sociales Vol II Universidad Iberoamericana / Universidad de los Lagos; 1999. 